\title{
A comparative study on the performance of meshless approximations and their integration
}

\author{
W. Quak • A. H. van den Boogaard • D. González • \\ E. Cueto
}

Received: 13 October 2010 / Accepted: 1 February 2011 / Published online: 4 March 2011

(C) The Author(s) 2011. This article is published with open access at Springerlink.com

\begin{abstract}
The goal of this research is to study the performance of meshless approximations and their integration. Two diffuse shape functions, namely the moving least-squares and local maximum-entropy function, and a linear triangular interpolation are compared using Gaussian integration and the stabilized conforming nodal integration scheme. The shape functions and integration schemes are tested on two elastic problems, an elasto-plastic problem and the inf-sup test. The elastic computation shows a somewhat lower accuracy for the linear triangular interpolation than for the two diffuse functions with the same number of nodes. However, the computational effort for this interpolation is considerably lower. The accuracy of the calculations in elasto-plasticity depends to great extend on the used integration scheme. All shape functions, and even the linear triangular interpolation, perform very well with the nodal integration scheme and locking-free behavior is shown in the inf-sup test.
\end{abstract}

Keywords Meshless methods - Nodal integration . Elasto-plasticity

\section{Introduction}

Nowadays, the finite element method is the first choice whenever a problem in solid mechanics needs to be simulated.

W. Quak $(\varangle) \cdot$ A. H. van den Boogaard Group of Mechanics of Forming Processes,

Faculty of Engineering Technology, University of Twente, PO Box 217, 7500 AE Enschede, The Netherlands

e-mail:W.Quak@utwente.nl

D. González · E. Cueto

Group of Structural Mechanics and Material Modelling, Aragón Institute of Engineering Research (I3A),

University of Zaragoza, Maria de Luna 5, 50018 Zaragoza, Spain
Nevertheless, performing a good finite element simulation can be difficult for certain problem types. Especially in large deformation processes, like for instance extrusion or injection molding, finite elements can suffer from excessive mesh distortion. Meshless methods are well suited to avoid these problems.

Many meshless methods have been developed during the last decades. The first development was the method of smooth particle hydrodynamics (SPH) as introduced by Lucy [33]. Thereafter similar methods employing diffuse approximations have been introduced; for instance the diffuse element method (DEM) by Nayroles et al. [34], the reproducing kernel particle method (RKPM) by Liu et al. [28-32], the point interpolation method (PIM) by $[25,43]$ and the element-free Galerkin method (EFG) by Belytschko et al. [6-8]. All these methods use a diffuse shape function to parameterize the displacement field in combination with a strong or weak form of equilibrium. For an extensive overview on the subject, containing most of the previously mentioned methods, the article of Li and Liu can be read [24]. A unifying stability analysis on these type of approximations is given in Belytschko et al. [5]. A meshless method with a less diffuse approximation for instance, is the natural element method (NEM) as proposed by Braun and Sambridge [10] or the particle finite element method (PFEM) by Idelsohn et al. [21,22]. The latter employs a finite element shape function in a meshless strategy. One of the latest developments in the field of meshless methods is the use of maximum-entropy principles to construct shape functions for use in solid mechanics. Examples are the maximum-entropy approximation (max-ent) by Sukumar $[40,41]$ or the modified, local maximum-entropy approximation (local max-ent) by Arroyo and Ortiz [2].

Since the introduction of meshless methods, numerical integration of the weak form has drawn considerable 
attention. The main reason for this attention is two-fold. First of all, the accurate integration of non-polynomial meshless shape functions is more involving than the integration of polynomial finite element approximations. The patch test for example, is not satisfied for a limited set of Gaussian integration points. Secondly, since numerical integration closely relates to volumetric locking for incompressible media, an integration rule has to be selected carefully for these media. Research on both aspects was done for instance for the element-free Galerkin method by Dolbow and Belytschko $[16,17]$ and by Askes et al. [3]. An integration scheme which overcomes the two previously mentioned problems is the stabilized conforming nodal integration scheme (SCNI) as proposed by Chen et al. [13,14]. This scheme satisfies the patch test without using an unfeasible amount of integration points and seems to be free of volumetric locking. The scheme has been investigated in the case of the natural element method by González et al. [18] and by Yoo et al. [44]. Similarly, applying this nodal integration to finite elements gives some interesting properties. The first concern, satisfying the patch test, is not an issue for classical compatible finite elements since this test is met by default. Volumetric locking on the contrary, seems to be avoided by applying this type of integration. The first development in this field is the nodal pressure tetrahedral as proposed by Bonet and Burton [9]. Afterwards, similar methods have been developed for instance by Dohrmann et al. [15], Pires et al. [36], Liu et al. [26,27], Krysl and Zhu [23], Puso and Solberg [38] and Hung et al. [20]. The stability of nodal integration for both meshless and finite element approximations was investigated by Puso et al. [37].

Some of the meshless methods as discussed in the preceding paragraphs are regarded as being 'truly' meshless methods. This classification indicates that the method is not depending on a mesh at all, neither for the construction of the shape functions, nor for evaluation of the equilibrium. Typically, methods using a strong-form of the equilibrium fall in this subcategory of meshless methods. Examples are for instance the methods of SPH and RKPM (strong-form variant). The majority of meshless methods however does not fall into this category. Although their shape functions are not defined upon a user-defined mesh, a mesh is used for the integration of the weak-form. The research as presented in this paper is restricted to the latter type of methods, since they allow for straightforward use of arbitrary material models. For strong-form methods, spatial gradients of the stress appear in the equations making the use of advanced material models, such as elasto-plasticity, more complicated.

The objective of this paper is to quantitatively examine the performance of meshless approximations and their numerical integration. To investigate this performance, four numerical tests are performed; two in elasticity, one in elasto-plasticity and one inf-sup or LBB test. As explained in preceding sections, the amount of meshless approximations proposed in literature is extensive. Therefore, for this study, a subset of three shape functions are chosen such that a representative view on meshless approximations is presented. The first shape function is the moving least-squares function. This function is one of the most commonly used approximations in the meshless field. Secondly, a recent development, namely the local maximum-entropy approximation, is included in the analysis. This approximation possesses similar properties to the moving least-squares approximation, although it can simplify the handling of boundary conditions. Finally a linear interpolation based on a Delaunay triangulation is included. This type of approximation is well known in the finite element method. The first two shape functions, the moving least-squares function and the local maximumentropy function are typical diffuse approximations. Their shape function is based on a domain of influence instead of a mesh. The latter shape function, the triangle interpolation, is a compact shape function. The number of neighbors for a node with this interpolation is usually smaller than is the case for diffuse approximations. Concerning the evaluation of the weak form, two numerical integration schemes will be tested. These are the stabilized conforming nodal integration scheme and a Gaussian integration scheme based on a Delaunay triangulation. Several combinations can be made by combining a shape function and an integration scheme. Using the triangular interpolation with a Gaussian integration scheme results in a linear triangular finite element [45]. Integrating the same shape function nodally will give a scheme as proposed by Dohrmann et al. [15]. The scheme as proposed by Chen et al. [13] is obtained by integrating a diffuse approximation nodally. All these combinations will be compared in this study.

The paper is organized as follows. Firstly, an introduction into the used shape functions and the integration schemes is given in Sect. 2. A short outline on the computer program as used for the analysis is presented. Section 3 gives the results of the numerical study on the performance of all combinations of shape functions and integration schemes. Afterwards the computational efficiency of the methods is compared. The conclusion is given in the last section.

\section{Governing equations}

\subsection{General formulations}

The starting point for the derivation of nodal equilibrium is the equation of equilibrium in the strong form:

$\nabla \cdot \boldsymbol{\sigma}+\mathbf{f}=0 \quad$ in $\Omega$

where $\sigma$ is the Cauchy stress tensor, $\mathbf{f}$ is a vector representing the body forces and $\Omega$ is the domain under consideration. 
The boundary conditions for the equilibrium equations are:

$\mathbf{u}=\overline{\mathbf{u}}$ on $\Gamma_{\mathrm{u}}$

$\sigma \cdot \mathbf{n}=\overline{\mathbf{t}} \quad$ on $\Gamma_{\mathrm{t}}$

where $\overline{\mathbf{u}}$ is a prescribed displacement on boundary $\Gamma_{\mathrm{u}}, \overline{\mathbf{t}}$ is a prescribed traction on the boundary $\Gamma_{\mathrm{t}}$ and $\mathbf{n}$ is the outward normal on the boundary. Applying a weighed residual formulation and using Galerkin's method, the strong equilibrium of Eq. (1) is weakened to obtain the nodal equilibrium:

$$
\begin{aligned}
\int_{\Omega} \mathbf{B}^{\mathrm{T}} \boldsymbol{\sigma} \mathrm{d} \Omega & =\int_{\Gamma_{\mathrm{t}}} \mathbf{N}^{\mathrm{T}} \overline{\mathbf{t}} \mathrm{d} \Gamma+\int_{\Omega} \mathbf{N}^{\mathrm{T}} \mathbf{f} \mathrm{d} \Omega \\
\mathbf{F}_{\text {int }} & =\mathbf{F}_{\text {ext }}
\end{aligned}
$$

where $\mathbf{F}_{\text {int }}$ is the internal force vector and $\mathbf{F}_{\text {ext }}$ is the external force vector. Matrices $\mathbf{N}$ and $\mathbf{B}$ relate the field displacements and strains to the nodal displacement vector $\mathbf{d}$ :

$\mathbf{u}=\mathbf{N d}$

$\varepsilon=\mathbf{B d}$

Matrix B contains the terms of the small strain tensor:

$\boldsymbol{\varepsilon}=\frac{1}{2}\left(\nabla \mathbf{u}+(\nabla \mathbf{u})^{\mathrm{T}}\right)$

The matrices $\mathbf{N}$ and $\mathbf{B}$ are constructed by using shape functions $\phi$. The formulations of the shape functions to construct the two matrices are given in Sect. 2.2. The integrator $\int_{\Omega} \ldots \mathrm{d} \Omega$ of Eq. (4) is worked out with two different numerical integration schemes as will be explained in Sect. 2.3.

For the constitutive equations a linear elastic model and an elasto-plastic model are used. If the latter model is used, the nodal equilibrium represented by Eq. 5 is found by a Newton-Rhapson iterative procedure. Within this procedure a prediction of the displacements is made by linearizing the internal force vector:

$$
\begin{aligned}
\mathbf{K} & =\frac{\partial \mathbf{F}_{\text {int }}}{\partial \mathbf{d}} \\
& =\int_{\Omega} \mathbf{B}^{\mathrm{T}} \mathbf{C B} \mathrm{d} \Omega
\end{aligned}
$$

where $\mathbf{K}$ is the stiffness matrix and $\mathbf{C}$ is the (algorithmic) material tangent matrix. In this research, a $\mathrm{J} 2$ radial return elasto-plastic material model is used. The formulation as well as the implementation of this model, including the algorithmic tangent and stress update, can be found for instance in Simo and Hughes [39]. The following set of equations is solved:

$\mathbf{K} \Delta \mathbf{d}^{k}=\mathbf{F}_{\mathrm{ext}}^{k}-\mathbf{F}_{\mathrm{int}}^{k}$

where $\Delta \mathbf{d}^{k}$ is a vector containing the iterative nodal displacement degrees of freedom and index $k$ denotes the current iteration step. The total nodal displacement degrees of freedom are found by summation of $\Delta \mathbf{d}^{k}$ over all iterations $k$.
2.2 Shape functions

Approximating the displacement field in case of meshless methods or the finite element method is done by a set of shape functions $\phi(\mathbf{x})$. The parameterized displacement field can be represented as:

$\mathbf{u}_{\mathrm{h}}(\mathbf{x})=\sum_{i=1}^{N_{\text {nod }}} \phi_{i}(\mathbf{x}) \mathbf{d}_{i}$

where $N_{\text {nod }}$ is the number of nodes in the model, and $\mathbf{u}_{\mathrm{h}}$ is the approximated displacement field. The vector $\mathbf{d}_{i}$ contains the nodal displacement degrees of freedom of node $i$ and is defined for $2 \mathrm{D}$ as follows:

$$
\left\{\mathbf{d}_{i}\right\}=\left\{\begin{array}{ll}
d_{x}^{i} & d_{y}^{i}
\end{array}\right\}^{\mathrm{T}}
$$

Although there is a lot of freedom in defining $\phi$, for the successful application of a shape function in solid mechanics two properties are essential. The first requirement for $\phi$, is the partition of unity:

$$
\sum_{i=1}^{N_{\text {nod }}} \phi_{i}(\mathbf{x})=1
$$

If the partition of unity is satisfied, rigid body translations can be represented exactly. The second property is known as first order reproducibility:

$$
\sum_{i=1}^{N_{\text {nod }}} \phi_{i}(\mathbf{x}) \mathbf{x}_{i}=\mathbf{x}
$$

Shape functions which satisfy this condition will reproduce a constant strain field exactly. In this study, three different types of shape functions are included that satisfy both conditions. Below, a short summary of their main formulations is presented. A full formulation can be found in the cited literature.

\subsubsection{Moving least-squares}

Moving least-squares approximations were firstly introduced in the field of computational solid mechanics by means of the diffuse element method by Nayroles et al. [34]. The starting point of the method is the assumption that the displacement field can be described locally by a polynomial:

$u_{\mathrm{h}}(\mathbf{x})=\mathbf{p}(\mathbf{x})^{\mathrm{T}} \mathbf{a}(\mathbf{x})$

where $\mathbf{p}(\mathbf{x})$ is a vector containing the components of a polynomial basis at the point $\mathbf{x}$, and $\mathbf{a}(\mathbf{x})$ is the corresponding set of coefficients at point $\mathbf{x}$. The parameters $\mathbf{a}(\mathbf{x})$ belonging to the polynomial basis $\mathbf{p}(\mathbf{x})$ are found by minimizing a potential expressing the residual between the approximated displacement field and the nodal displacements $d_{i}$, similarly 
to a 'normal' least squares fit. This potential is defined as:

$\Pi_{\mathrm{mls}}(\mathbf{x})=\sum_{i=1}^{N_{\text {nod }}} \omega\left(\mathbf{x}-\mathbf{x}_{i}\right)\left(\mathbf{p}\left(\mathbf{x}_{i}\right)^{\mathrm{T}} \mathbf{a}(\mathbf{x})-d_{x}^{i}\right)^{2}$

In this paper the following polynomial is used:

$\mathbf{p}(\mathbf{x})=\left\{\begin{array}{lll}1 & x & y\end{array}\right\}^{\mathrm{T}}$

The weight function in $2 \mathrm{D}$ is constructed by multiplying two 1D functions $\omega_{1}$ :

$\omega\left(\mathbf{x}-\mathbf{x}_{i}\right)=\omega_{1}\left(\frac{x-x_{i}}{\bar{h}}\right) \omega_{1}\left(\frac{y-y_{i}}{\bar{h}}\right)$

For this weight function $\omega_{1}$, a cubic spline is chosen:

$\omega_{1}(s)= \begin{cases}\frac{2}{3}-4 s^{2}+4 s^{3} & \text { for } s \leqslant \frac{1}{2} \\ \frac{4}{3}-4 s+4 s^{2}-\frac{4}{3} s^{3} & \text { for } \frac{1}{2}<s \leqslant 1 \\ 0 & \text { for } s>1\end{cases}$

where $s$ is a local coordinate. Parameter $\bar{h}$ is found by considering $\bar{h}=h \cdot d$, where $h$ is an average measure of the spacing of nodes and $d$ controls the relative size of the domain of influence of the shape function. For the current analysis, $\bar{h}$ is chosen to be equal for all nodes. In a regularly spaced grid, $h$ is equal to the minimum distance between two neighboring nodes. If the value of parameter $d$ is increased, the shape functions become more diffuse.

For a certain location $\mathbf{x}$, the potential $\Pi_{\mathrm{mls}}(\mathbf{x})$ is minimized with respect to parameters $\mathbf{a}(\mathbf{x})$ :

$$
\begin{aligned}
\mathbf{a}(\mathbf{x})= & \left(\sum_{i=1}^{N_{\text {nod }}} \omega\left(\mathbf{x}-\mathbf{x}_{i}\right) \mathbf{p}\left(\mathbf{x}_{i}\right) \mathbf{p}\left(\mathbf{x}_{i}\right)^{\mathrm{T}}\right)^{-1} \\
& \cdot \sum_{i=1}^{N_{\text {nod }}} \omega\left(\mathbf{x}-\mathbf{x}_{i}\right) \mathbf{p}\left(\mathbf{x}_{i}\right) d_{x}^{i}
\end{aligned}
$$

Substitution of $\mathbf{a}(\mathbf{x})$ into Eq. (16) gives an expression for $\boldsymbol{\phi}(\mathbf{x})$. The minimization of Eq. (21) must be performed for location $\mathbf{x}$ at which the shape functions values or gradients are required. The MLS approximation has a continuity of degree 2.

\subsubsection{Local maximum-entropy}

Local maximum-entropy shape functions were recently introduced by Arroyo and Ortiz [2] and use informationtheoretic principles to approximate the displacement field. The approximation is constructed by considering the probability $\phi$ that a nodal value holds at an arbitrary point in space. A potential containing both an entropy term related to this probability, as well as a potential expressing the locality of this probability distribution is constructed. This potential is formulated as:

$\Pi_{1 \mathrm{me}}=\beta U(\mathbf{x}, \boldsymbol{\phi})-H(\boldsymbol{\phi})$ with the Shannon entropy $H$ defined as:

$H(\boldsymbol{\phi})=\sum_{i=1}^{N_{\text {nod }}} \phi_{i} \ln \left(\phi_{i}\right)$

and the locality function:

$U(\mathbf{x}, \boldsymbol{\phi})=\sum_{i=1}^{N_{\text {nod }}} \phi_{i}\left\|\mathbf{x}-\mathbf{x}_{i}\right\|^{2}$

In the potential $\Pi_{l m e}$, the parameter $\beta$ is used to control the compactness of the approximation. This parameter is related to the average spacing of nodes $h$ and a parameter $\gamma$ as follows:

$\beta=\frac{\gamma}{h^{2}}$

By setting $\gamma$ either compact or diffuse shape functions can be obtained. Shape functions $\phi$ are found by minimizing Eq. (22). This minimization is constrained by Eqs. (14) and (15). For a detailed description of this minimization the reader is referred to Arroyo and Ortiz [2].

An interesting aspect of local maximum-entropy shape functions is its behavior at the boundary. Shape functions of nodes on the convex hull possess the Kronecker delta property, and shape functions of internal nodes have a value of zero at the convex hull. Nonetheless, this characteristic does not prevent the shape functions from being diffuse internally. Here, the LME approximation possesses $C^{\infty}$ continuity.

\subsubsection{Linear triangle interpolation}

LME approximations with a high $\gamma$ value result in a linear interpolation on Delaunay triangles (excluding degenerate cases). For this reason also an explicit linear triangular shape function is considered. This type of approximation is well known in finite element analysis and expressions can be found in most books on finite element technology; for instance in Zienkiewicz and Taylor [45]:

$\left\{\begin{array}{l}\phi_{1}(\mathbf{x}) \\ \phi_{2}(\mathbf{x}) \\ \phi_{3}(\mathbf{x})\end{array}\right\}=\left[\begin{array}{ccc}x_{1} & x_{2} & x_{3} \\ y_{1} & y_{2} & y_{3} \\ 1 & 1 & 1\end{array}\right]^{-1}\left\{\begin{array}{l}x \\ y \\ 1\end{array}\right\}$

where $x_{i}$ and $y_{i}$ are the coordinates of node $i$. The most profound argument to choose this type of shape function is its low computational cost and its simple formulation. Another advantage is the straightforward imposition of boundary conditions since it possesses the Kronecker-delta property for all nodes in the domain. The order of continuity is $C^{0}$, hence smooth strain fields are not obtained for limited sets of nodes. 


\subsection{Integration schemes}

In this research, two integration schemes are used to evaluate Eqs. (4) and (10) numerically. The first scheme is the well known 'Gauss' integration scheme as is commonly used in finite elements. The second scheme is the stabilized conforming nodal integration scheme, also known by the abbreviation of SCNI. Both schemes are shortly explained below.

\subsection{1 'Gauss' integration}

'Gauss' integration of the internal force vector is formulated as follows:

$$
\begin{aligned}
\mathbf{F}_{\text {int }} & =\int_{\Omega} \mathbf{B}^{\mathrm{T}} \boldsymbol{\sigma} \mathrm{d} \Omega \\
& \approx \sum_{k=1}^{N_{\text {int }}} \mathbf{B}^{\mathrm{T}}\left(\mathbf{x}_{k}\right) \boldsymbol{\sigma}\left(\mathbf{x}_{k}\right) \Delta \Omega_{k}
\end{aligned}
$$

$N_{\text {int }}$ is the total number of integration points in the body and $\mathbf{x}_{k}$ is the location of an integration point. Usually the summation $\sum_{k=1}^{N_{\text {int }}}$ is split into a sum over elements or integration cells and over integration points for such an element or cell. In this research an integration rule within a triangle is used. The starting point is a cloud of nodes which is triangulated by means of a Delaunay triangulation. Within each triangle an integration rule is defined.

If, for finite elements, the integration rule is chosen in accordance with the interpolation functions, the patch test is satisfied. Details on this test can be found for instance in Zienkiewicz and Taylor [45]. Note that non-polynomial meshless approximations in general do not pass the patch test, even if these approximations possess first order reproducibility. Inexact integration precludes in this case an exact evaluation of the linear displacement field.

\subsubsection{Nodal integration}

Nodal integration of the internal force vector can be expressed as:

$$
\begin{aligned}
\mathbf{F}_{\text {int }} & =\int_{\Omega} \mathbf{B}^{\mathrm{T}} \boldsymbol{\sigma} \mathrm{d} \Omega \\
& \approx \sum_{i=1}^{N_{\text {nod }}} \mathbf{B}^{\mathrm{T}}\left(\mathbf{x}_{i}\right) \boldsymbol{\sigma}\left(\mathbf{x}_{i}\right) \Delta \Omega_{i}
\end{aligned}
$$

where $N_{\text {nod }}$ is the number of nodes, $\mathbf{x}_{i}$ is the location of a node and $\Delta \Omega_{i}$ is the volume accompanying that particular node. Matrix $\mathbf{B}$ is the strain-displacement matrix which is consistent with the displacement field. Several problems arise when using an integration scheme according to Eq. (30). The two main problems are the lack of stability and the loss of first

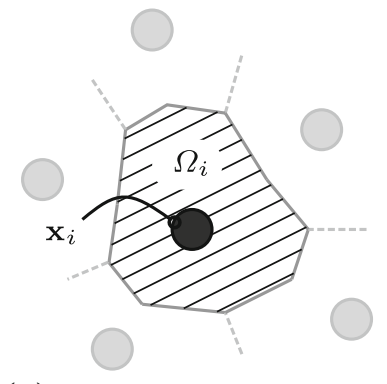

(a) volume integration according to Equation (31)

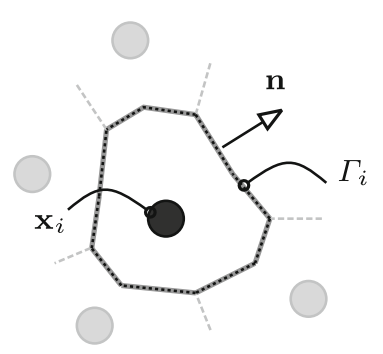

(b) contour integration according to Equation (32)
Fig. 1 Cell integration for SCNI

order reproducibility in case of non-polynomial approximations. An improved nodal integration scheme was proposed by Chen et al. [13]. This stabilized conforming nodal integration scheme (SCNI) modifies the definition of $\mathbf{B}$ in order to avoid these problems. The essence of the method is that an assumed displacement gradient at a node is constructed by averaging the displacement gradient over a cell accompanying that node:

$\tilde{\nabla} \mathbf{u}\left(\mathbf{x}_{i}\right)=\frac{1}{\Omega_{i}} \int_{\Omega_{i}} \nabla \mathbf{u} \mathrm{d} \Omega$

The volume integral can be rewritten by means of the Gauss divergence theorem to a surface integral:

$\tilde{\nabla} \mathbf{u}\left(\mathbf{x}_{i}\right)=\frac{1}{\Omega_{i}} \int_{\Gamma_{i}} \mathbf{n} \mathbf{u} \mathrm{d} \Gamma$

where $\mathbf{n}$ is the outward normal on boundary $\Gamma_{i}$ of the cell $\Omega_{i}$. The assumed strain field used for the integration becomes:

$\tilde{\boldsymbol{\varepsilon}}\left(\mathbf{x}_{i}\right)=\frac{1}{2}\left(\tilde{\nabla} \mathbf{u}\left(\mathbf{x}_{i}\right)+\left(\tilde{\nabla} \mathbf{u}\left(\mathbf{x}_{i}\right)\right)^{\mathrm{T}}\right)$

Figure 1 gives an illustration on the nodal integration scheme. Section 2.5 gives a description on the construction of the cells as displayed in Fig. 1. The modified $\mathbf{B}$-matrix at node $\mathbf{x}_{i}$ in $2 \mathrm{D}$ becomes:

$\left[\begin{array}{ll}\tilde{\mathbf{B}}\left(\mathbf{x}_{i}\right)\end{array}\right]=\left[\begin{array}{llll}\tilde{\mathbf{B}}_{1}\left(\mathbf{x}_{i}\right) & \tilde{\mathbf{B}}_{2}\left(\mathbf{x}_{i}\right) & \ldots & \tilde{\mathbf{B}}_{N_{\mathrm{nod}}}\left(\mathbf{x}_{i}\right)\end{array}\right]$

where the contribution of shape function $j$ is defined as:

$$
\left[\tilde{\mathbf{B}}_{j}\left(\mathbf{x}_{i}\right)\right]=\frac{1}{\Delta \Omega_{i}} \int_{\Gamma_{i}}\left[\begin{array}{cc}
\phi_{j} n_{1} & 0 \\
0 & \phi_{j} n_{2} \\
\phi_{j} n_{2} & \phi_{j} n_{1}
\end{array}\right] \mathrm{d} \Gamma
$$

The resulting internal force vector becomes:

$\mathbf{F}_{\text {int }}=\sum_{i=1}^{N_{\text {nod }}} \tilde{\mathbf{B}}^{\mathrm{T}}\left(\mathbf{x}_{i}\right) \tilde{\boldsymbol{\sigma}}\left(\mathbf{x}_{i}\right) \Delta \Omega_{i}$ 
where $\tilde{\boldsymbol{\sigma}}\left(\mathbf{x}_{i}\right)$ is the stress tensor computed with the assumed strain $\tilde{\boldsymbol{\varepsilon}}\left(\mathbf{x}_{i}\right)$. The stiffness matrix becomes:

$$
\mathbf{K}=\sum_{i=1}^{N_{\text {nod }}} \tilde{\mathbf{B}}^{\mathrm{T}}\left(\mathbf{x}_{i}\right) \mathbf{C} \tilde{\mathbf{B}}\left(\mathbf{x}_{i}\right) \Delta \Omega_{i}
$$

An interesting aspect of integrating the weak equations nodally is that a material point is at the location of the node. Especially if more sophisticated, history dependent material models are used, all data concerning the material model can be stored at the location of the node. This can simplify for instance re-meshing or convecting algorithms, such that the spatial distribution of the state variables of the material model is optimally preserved. Note that due to the nodal integration, the connectivity increases. All shape functions holding a nonzero value in $\Omega_{i}$ contribute to $\tilde{\mathbf{B}}\left(\mathbf{x}_{i}\right)$. Matrix $\mathbf{K}$ will therefore become less sparse.

\subsection{Applying boundary conditions}

Applying the boundary conditions in case of meshless shape functions requires a different approach than usually used for finite elements. For finite elements the Kronecker Delta property holds, which implies that the field displacement at the position of the node is equal to that nodal displacement:

$\mathbf{u}_{\mathrm{h}}\left(\mathbf{x}_{i}\right)=\mathbf{d}_{i}$

where $\mathbf{x}_{i}$ is the nodal location. Most meshless approximations do not satisfy this property. Displacements cannot be enforced by simply prescribing entries in the nodal displacement vector $\mathbf{d}$. In this paper, the method of Lagrangian multipliers is applied to enforce prescribed boundary displacements similarly as was done by Belytschko et al. [8]. The set of nodal degrees of freedom is expanded by a set of Lagrangian multipliers. The system of equations with the added degrees of freedom becomes:

$$
\left[\begin{array}{cc}
\mathbf{K} & \mathbf{G}^{\mathrm{T}} \\
\mathbf{G} & \mathbf{0}
\end{array}\right]\left\{\begin{array}{c}
\Delta \mathbf{d}_{i} \\
\Delta \lambda_{i}
\end{array}\right\}=\left\{\begin{array}{c}
\mathbf{F}_{\mathrm{ext}}^{i}-\mathbf{F}_{\text {int }}^{i} \\
\mathbf{g}
\end{array}\right\}
$$

where $\Delta \lambda_{i}$ is a vector containing the Lagrangian multipliers and matrix $\mathbf{K}$ is the stiffness matrix as defined in Eq. (10) or Eq. (37) depending on the integration scheme. Vector $\mathbf{g}$ and matrix $\mathbf{G}$, are defined as:

$$
\begin{gathered}
\mathbf{g}=-\int_{\Gamma} \mathbf{N}_{\lambda}^{\mathrm{T}} \overline{\mathbf{u}} \mathrm{d} \Gamma \\
\mathbf{G}=-\int_{\Gamma} \mathbf{N}_{\lambda}^{\mathrm{T}} \mathbf{N} \mathrm{d} \Gamma
\end{gathered}
$$

Eqs. (40) and (41) are evaluated with a nodal integration rule as used by Pannachet and Askes [35]. Furthermore, the shape functions related to the Lagrangian multipliers are chosen to have the Kronecker delta property. As a result only displacement related shape functions need to be evaluated since $\mathbf{N}_{\lambda}^{i}\left(\mathbf{x}_{j}\right)=\delta_{i j}$.

The boundary conditions for the INT function are enforced by using a simple row reduction technique as is standard in finite element analysis. Prescribed displacements are multiplied with corresponding columns in $\mathbf{K}$ and added to the right-hand side of Eq. (11).

\subsection{Triangulations and tesselations}

Both integration schemes as well as the linear interpolation function require geometrical objects. The SCNI integration scheme uses a tessellation to construct the modified strain matrix $\tilde{\mathbf{B}}$ and the Gaussian integration scheme is defined upon a triangle. Moreover, this triangulation is beneficial for the MLS and LME shape functions as well, since this data structure can be used for efficient neighbor searching. Note that the terminology 'meshless method' only refers to the shape function and not to the integration cells for instance.

The strategy to obtain the geometrical objects is as follows. First of all, a cloud of nodes is triangulated with a Delaunay triangulation. In two-dimensions, the corners of a Delaunay triangle are those three nodes that share a circumscribed circle not containing any other node of the cloud of nodes. Afterwards, the concept of $\alpha$-shapes ensures that concave boundaries can be represented without defining that concave boundary explicitly. A description of the method for solid mechanics can be found in Alfaro et al. [1]. For the SCNI integration, a set of cells is made with a technique as used by Chen et al. [14]. It takes the midpoints of the sides of a Delaunay triangle and the centroid of that triangle. The cell of a node is made by connecting straight lines through these points for all triangles connected to that particular node. The main benefit of constructing cells in this fashion in comparison with the well known Voronoi tesselation is that boundaries can be tesselated more easily. Moreover, if the Delaunay triangulation is known, this tesselation can be constructed with little extra effort. Figure 2 gives a visualization of the geometrical objects as described above.

\subsection{Overview of the implementation}

To switch easily between shape function, integration scheme, or method to apply boundary conditions, a code was written of which the architecture will be shortly outlined here.

Figure 3 displays a flowchart of the computer program. The components named MLS, LME and INT are abbreviations of moving least-squares, local maximum-entropy and linear triangular interpolation, respectively. The Galerkin weak form is integrated with the two integration schemes. These are the standard 'Gaussian' integration scheme and the stabilized conforming nodal integration scheme abbreviated 


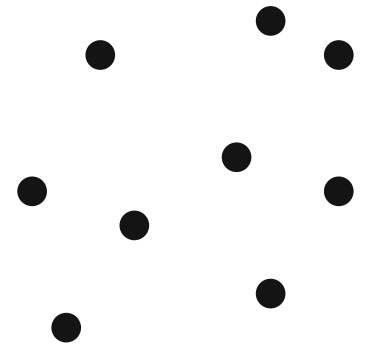

(a) cloud of nodes

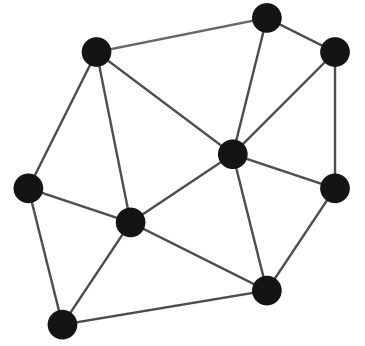

(b) triangulation

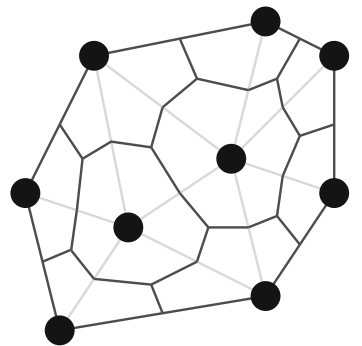

(c) tesselation

Fig. 2 Geometrical objects used for the analysis

with STD and SCNI, respectively. From a programming point of view, the main difference between these two integration rules is that the STD integration consists of a loop over triangles for the stiffness matrix and internal force vector and the SCNI integration loops over nodes for the assembly procedure. Furthermore, the STD integration requires the gradients of the shape functions whereas for the SCNI integration, just the shape function values are required. The averaged derivatives follow from the divergence theorem.

The method to apply the boundary conditions is selected depending on which shape function is chosen. Lagrangian multipliers will be used for the LME and MLS functions. For the INT function the row reduction technique is employed, resulting in the reduced stiffness matrix $\mathbf{K}_{\text {red}}$.

\section{Numerical performance}

\subsection{Introduction}

In this section the numerical performance of the shape functions and integration schemes will be examined. First of all, a

$$
\begin{gathered}
y, 000 \\
0,02000000 \\
00000
\end{gathered}
$$

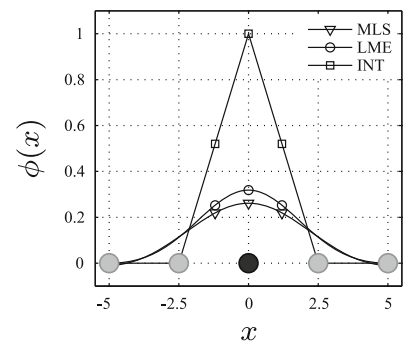

Fig. 4 Shape functions as used in the analysis

test in linear elasticity is performed to examine the accuracy and convergence properties of the shape functions and integration schemes. An infinite plate with a hole is used as test problem. Secondly, an analysis is done to examine the performance of various combinations in elasto-plasticity. The effect of the type of integration, the order of the Gaussian integration and the compactness of the diffuse approximations will be investigated. Finally the computational efficiency of the implementation will be assessed.

For the MLS and LME approximations, parameters $d$ and $\gamma$ have to be set. To make a fair comparison between these approximations in the following tests, the parameters are set such that the shape functions have a similar domain of influence. For the MLS approximation $d=2.6$, and for the LME approximation $\gamma=1$, unless stated otherwise. Figure 4 gives a visualization of the shape functions with these parameters. These settings for the domain of influence were found to give a stable response in most cases. Making the shape functions too compact for instance, can result in failure of the shape function algorithm.

\subsection{Plate with a hole problem}

In this test the accuracy and convergence of all combinations of shape functions and integration schemes is tested on the problem of an infinite plate with a hole. Figure 5 shows the geometry of the infinite plate at the left-hand side, and the modeled part of the plate at the right-hand side. The infinite plate is loaded in horizontal direction with a uniform traction. The geometry is simplified by using symmetry conditions and only evaluating a small part of the plate close to the location of the hole. At the symmetry lines the appropriate displacement boundary conditions are imposed, and
Fig. 3 Schematic representation of the program as implemented in MATLAB

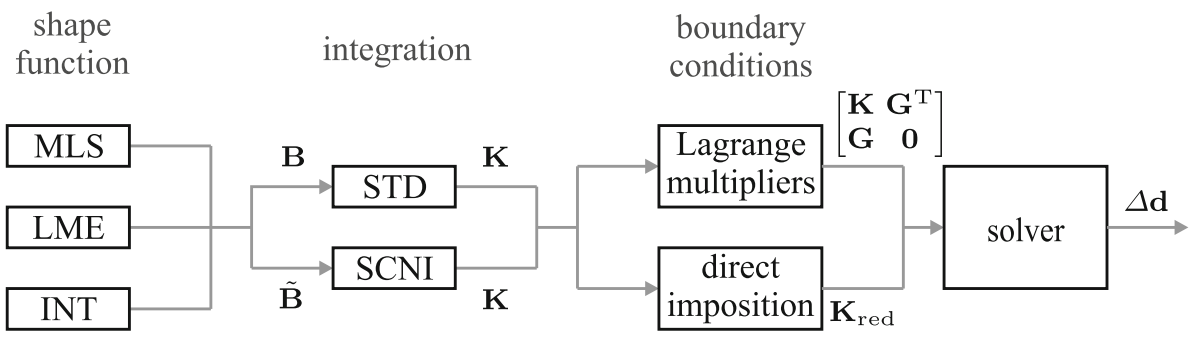



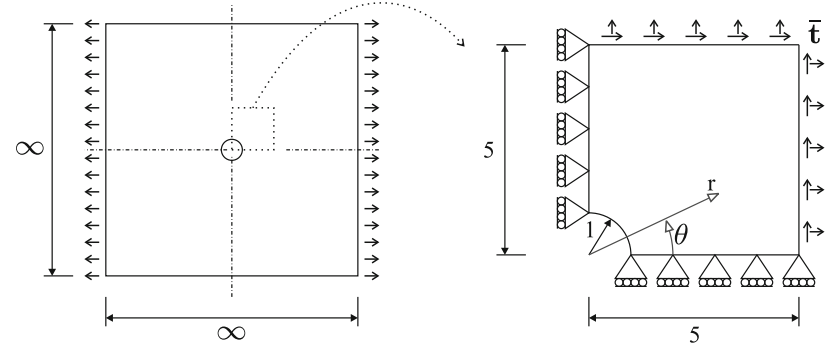

Fig. 5 Geometry of the infinite plate with a hole problem

at the free boundary the known exact stress field is applied. The exact solution of the problem can be found for instance in Timoshenko and Goodier [42]. The exact stress field is:

$\sigma_{x x}(r, \theta)=1-\frac{1}{r^{2}}\left(\frac{3}{2} \cos 2 \theta+\cos 4 \theta\right)+\frac{3}{2 r^{4}} \cos 4 \theta$

$\sigma_{y y}(r, \theta)=-\frac{1}{r^{2}}\left(\frac{1}{2} \cos 2 \theta-\cos 4 \theta\right)-\frac{3}{2 r^{4}} \cos 4 \theta$

$\sigma_{x y}(r, \theta)=-\frac{1}{r^{2}}\left(\frac{1}{2} \sin 2 \theta+\sin 4 \theta\right)+\frac{3}{2 r^{4}} \sin 4 \theta$

and the corresponding displacement field is:

$$
\begin{aligned}
u_{x}(r, \theta)= & \frac{1}{8 \mu}(r(\kappa+1) \cos \theta \\
& \left.+\frac{2}{r}((1+\kappa) \cos \theta+\cos 3 \theta)-\frac{2}{r^{3}} \cos 3 \theta\right) \\
u_{y}(r, \theta)= & \frac{1}{8 \mu}(r(\kappa-3) \sin \theta \\
& \left.+\frac{2}{r}+((1-\kappa) \sin \theta+\sin 3 \theta)-\frac{2}{r^{3}} \sin 3 \theta\right)
\end{aligned}
$$

Parameter $\kappa$ and $\mu$ are defined for plane strain as:

$\kappa=3-4 v$

$\mu=\frac{E}{2(1+v)}$

The Young's modulus and the Poisson's ratio are $E=10$ and $v=0.3$, respectively. For the STD integration scheme a 3 point integration rule within a triangle is used. The SCNI integration scheme employs a 2 point Gauss rule on each of the facets of a cell. To compare the accuracy of a combination of shape function and integration scheme, an error norm on the displacement is used. The error norm is a discrete version of the $\|\cdot\|_{\mathrm{L} 2}$-norm and samples the displacement error only at the nodes. This norm is used to avoid the problem of introducing errors in the computation of the integrand of the $\|\cdot\|_{\text {L2 }}$ error norm as was pointed out by González et al. [18].

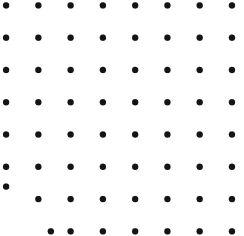

(a) coarse

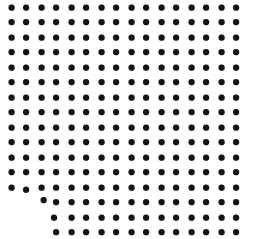

(b) medium

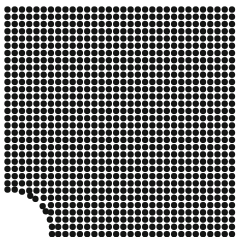

(c) fine
Fig. 6 Nodal grids for the plate with hole problem

The error is given by:

$\left\|e_{\mathrm{u}}\right\|_{2}=\frac{1}{N_{\text {nod }}} \sqrt{\sum_{i=1}^{N_{\text {nod }}}\left\|\mathbf{u}_{\mathrm{h}}\left(\mathbf{x}_{i}\right)-\mathbf{u}_{\text {exact }}\left(\mathbf{x}_{i}\right)\right\|^{2}}$

The location of a node is given by $\mathbf{x}_{k}$, the approximated solution is $\mathbf{u}_{\mathrm{h}}$, and $N_{\text {nod }}$ is the total number of nodes.

Figure 6 shows three nodal grids from coarse to fine as used for this test. The amount of nodes for the grids in the order of coarse to fine are 63, 248 and 993, respectively.

Figure 7 shows the $\left\|e_{\mathrm{u}}\right\|_{2}$ error for the integration schemes and the three shape functions. First of all, when comparing the shape functions it can be seen that regardless of the integration scheme used, the two diffuse approximations are more accurate than the linear interpolation. The error norms for the MLS and LME shape functions are two to three times smaller than the the INT function. The error for the MLS and LME approximation is found to be nearly identical due to the selection of parameters $\gamma$ and $d$. Secondly, the two integration schemes give approximately the same accuracy and rate of convergence for a specific shape function. Compared to the Gaussian integration, integrating the INT function with the SCNI scheme increases the accuracy.

\subsection{Distortion analysis}

In the following test, the approximations are tested on their behavior on distorted grids. The problem used to analyze this behavior is the pure bending of a square piece. The details are given in Fig. 8. At the right-hand side of the square, a traction in $x$-direction is prescribed varying linearly between 7.5 and -7.5 . The problem is discretized by five different nodal grids as shown in Fig. 9. Both small irregularities as well as substantial distortions are applied to a regular grid with increasing severity. The energy error norm is monitored over the five grids. This norm is defined as:

$\left\|e_{\mathrm{u}}\right\|_{\mathrm{E}}^{2}=\int_{\Omega}\left(\boldsymbol{\varepsilon}-\boldsymbol{\varepsilon}_{\mathrm{h}}\right)^{\mathrm{T}} \mathbf{C}\left(\boldsymbol{\varepsilon}-\boldsymbol{\varepsilon}_{\mathrm{h}}\right) \mathrm{d} \Omega$

where $\boldsymbol{\varepsilon}_{\mathrm{h}}$ and $\boldsymbol{\varepsilon}$ are the approximated strain field and the exact strain field respectively. The exact stress field of the problem can be found by simply considering the prescribed traction 


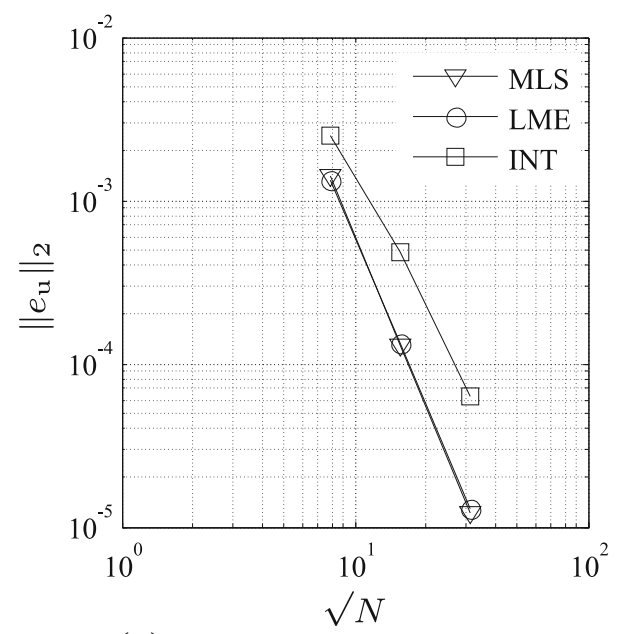

(a) STD Gaussian integration

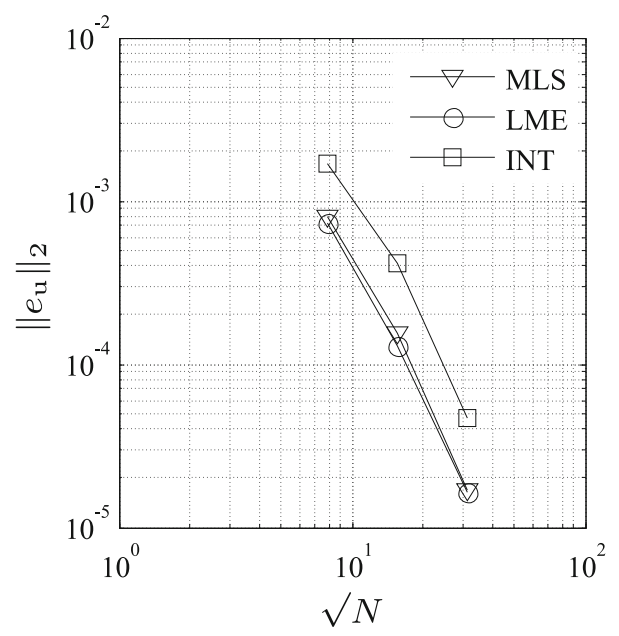

(b) SCNI integration

Fig. $7\left\|e_{\mathrm{u}}\right\|_{2}$ error norm for the plate with hole problem

on the right-hand side of the square piece of material:

$$
\begin{aligned}
& \sigma_{x x}(\mathbf{x})=\frac{3}{2} \cdot y \\
& \sigma_{y y}(\mathbf{x})=0 \\
& \sigma_{x y}(\mathbf{x})=0
\end{aligned}
$$

By using the constitutive behavior, the exact strain field of the problem can be found:

$$
\begin{aligned}
& \varepsilon_{x x}(\mathbf{x})=\frac{3 E(1-v)}{2(1+v)(1-2 v)} \cdot y \\
& \varepsilon_{y y}(\mathbf{x})=\frac{3 E v}{2(1+v)(1-2 v)} \cdot y \\
& \varepsilon_{x y}(\mathbf{x})=0
\end{aligned}
$$

The integral in Eq. (50) will be evaluated by the two integration schemes given in Sect. 2.3.1 and Sect. 2.3.2 depending on which integration scheme is used for the approximation.

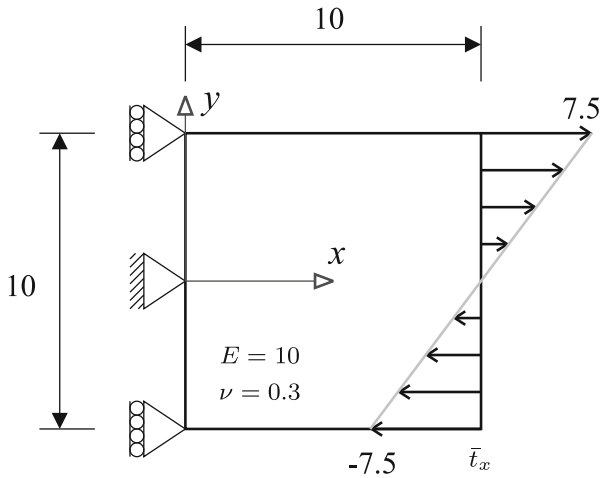

Fig. 8 Model to examine distortional effects

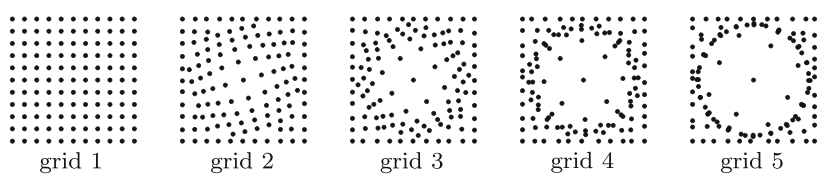

Fig. 9 Grids used to examine the influence of distortion

Figure 10 shows the results for the two integration schemes and the three shape functions. Several conclusions can be drawn from the figures. First of all, for low amounts of distortion (grid 1 and 2), the most accurate results are obtained by employing a MLS or LME shape function with a Gaussian integration scheme. Increasing the amount of distortion for the Gaussian integration scheme increases the error in the energy norm. For the last grids, problems were encountered in the construction of the MLS and LME shape functions. For the MLS functions for instance, parameters $\mathbf{a}(\mathbf{x})$ cannot be determined uniquely for certain locations $\mathbf{x}$. The domain of influence of these shape functions has to be increased, such that sufficient neighbors are present to define these shape functions properly. The Gaussian integrated triangular interpolation is inaccurate both at the regular grid as well as on the irregular grids.

The SCNI integrated solutions seem to be less affected by the distortion in general. Although the MLS function on grid 4 is found to be inaccurate, overall a smaller influence of the distortion is found. Similar to the Gaussian integrated solutions, problems were encountered with the MLS and LME functions on grid 4 and 5. It can be seen that the performance of the INT function improves considerably by employing the SCNI integration.

\subsection{Tapered bar analysis}

In this section the performance of the shape functions and integration schemes is investigated in an elasto-plastic analysis. Two potential problems can be envisaged for a numerical method in plasticity. Firstly, there is the problem of volumetric locking. If a numerical scheme suffers from volumetric 


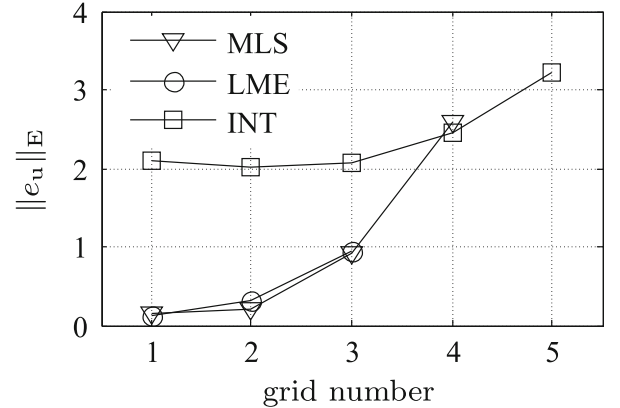

(a) STD Gaussian integration

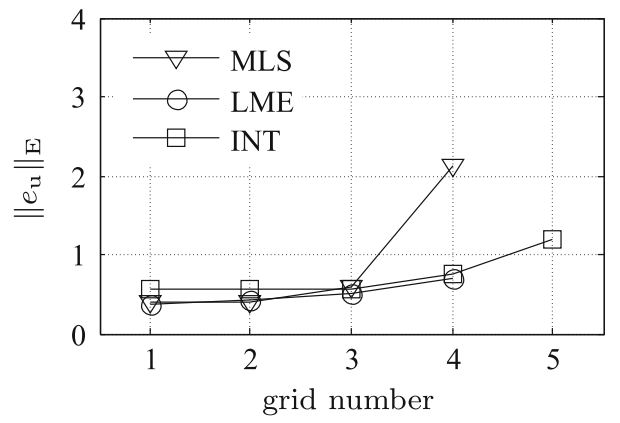

(b) SCNI integration

Fig. 10 Energy error norm with increasing distortion

locking, the response will become spuriously stiff in plastic deformation and the pressure associated with this deformation is nonphysical. Secondly, the transition from elastic to plastic will lead to a locally high gradient in the strain field. This interface should be distinct and not nonphysically diffuse as one might expect with diffuse meshless approximations.

Figure 11a shows the model used for the analysis. The tapered bar is loaded at the right-hand side with a prescribed traction. The problem is simplified by assuming a plane strain state, and symmetry along the center line of the bar. A VonMises yield criterion is used in combination with linear hardening:

$\sigma_{\mathrm{f}}=\sigma_{0}+C \varepsilon_{\mathrm{eq}}$

Variables $\sigma_{\mathrm{f}}$ and $\varepsilon_{\mathrm{eq}}$ are the flow stress and equivalent plastic strain, respectively. The constants for the hardening law are
$C=100$ and $\sigma_{0}=100$. The Young's modulus and Poisson's ratio of the elastic part are $E=210000$ and $v=0.3$, respectively. After applying the total load of 120 on the bar, the right-hand side of the bar will have deformed plastically, whereas the left-hand side is still in the elastic domain. Hence it should be possible to observe an elastic-plastic transition region within the bar. Due to the non-linear material response, Eq. (5) is solved with an iterative-incremental strategy. The stress update is performed with a fully implicit return mapping algorithm.

For the STD integration a three point and a one point integration rule is used. For the SCNI integration a two point Gauss rule is used on each of the facets of the cell. The nodal grid for the meshless approximations is shown in Fig. 11b. The grid has 4 nodes over the height and 11 along the length. The results of the meshless analysis are compared to a reference finite element solution. A dense mesh of linear quadrilateral selective reduced integrated (SRI) finite elements are used to get an accurate prediction of the stress and strain fields. This mesh has 14 elements over the height and 40 along the length and is shown in Fig. 11c.

\subsubsection{Influence of integration}

The first test is the simulation of the tapered bar problem with all shape functions and the two integration schemes. For the STD integration scheme a three point and a one point integration rule are used. The strain component $\varepsilon_{x x}$ is monitored along the symmetry line $(y=0)$. Figure 12 shows the results for the three cases where the horizontal axis displays the $x$ coordinate along the line of symmetry.

Figure 12a shows the results with the 3 point STD integration rule. It can be seen that the plastic deformation at the right-hand side of the bar is underestimated by the linear interpolation shape function. Due to the incompressibility of this plastic deformation, the numerical artefact of volumetric locking is deteriorating the results. Since a linear triangular shape function in combination with Gaussian integration rule makes a linear triangular finite element, this poor behavior in incompressibility is expected. The MLS and LME approximations are underestimating the strain $\varepsilon_{x x}$ slightly but are

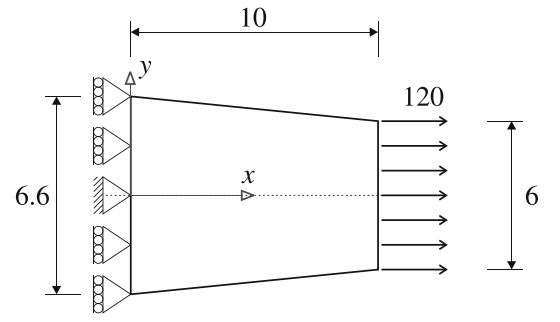

(a) model

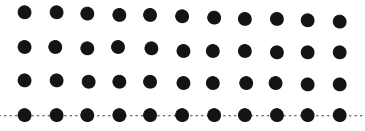

(b) cloud of nodes

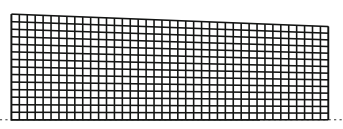

(c) quadrilateral mesh

Fig. 11 The tapered bar problem and the models for the meshless and reference finite element computations 


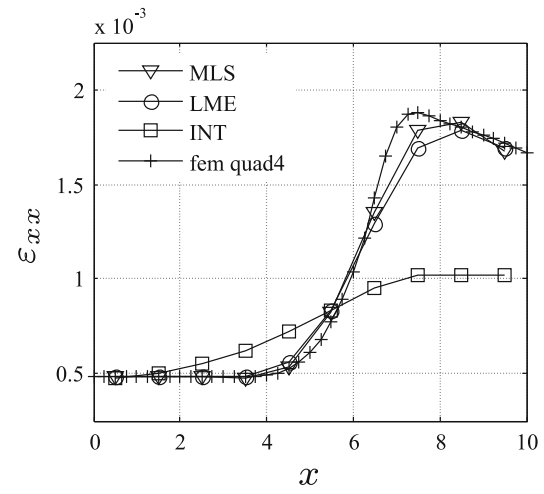

(a) STD integration (3 ip/triangle)

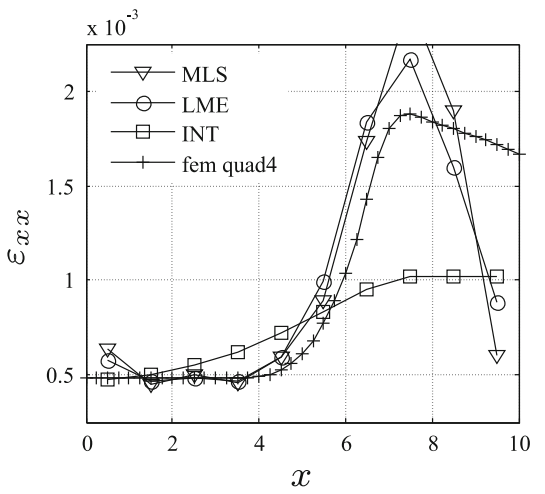

(b) STD integration (1 ip/triangle)

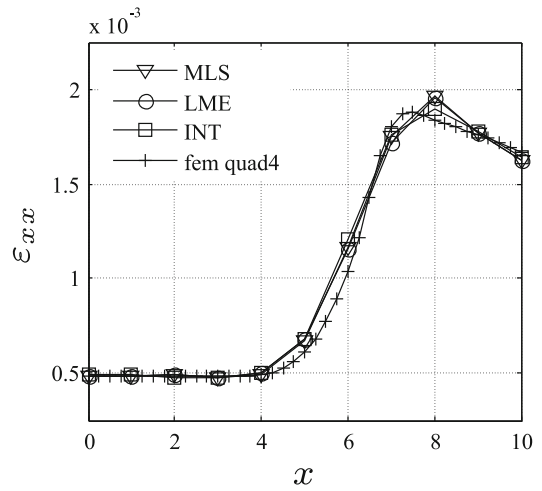

(c) SCNI integration

Fig. 12 Strain in $x$ direction along the symmetry axis

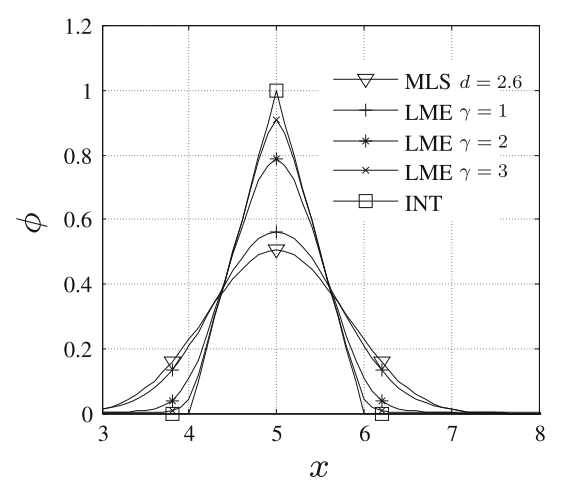

(a) Shape functions of the node at location $(x, y)=(5,0)$

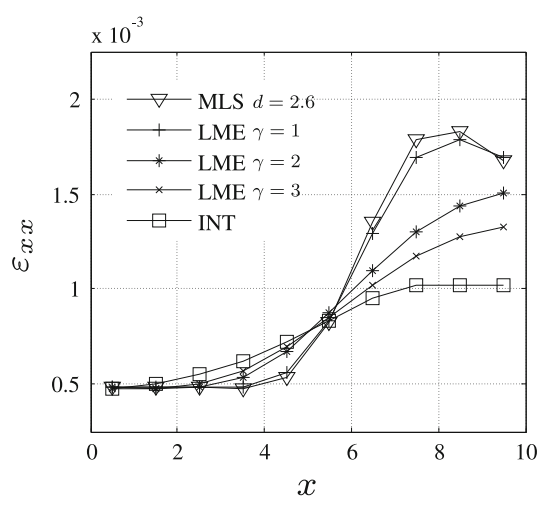

(b) STD integration (3 ip/triangle)

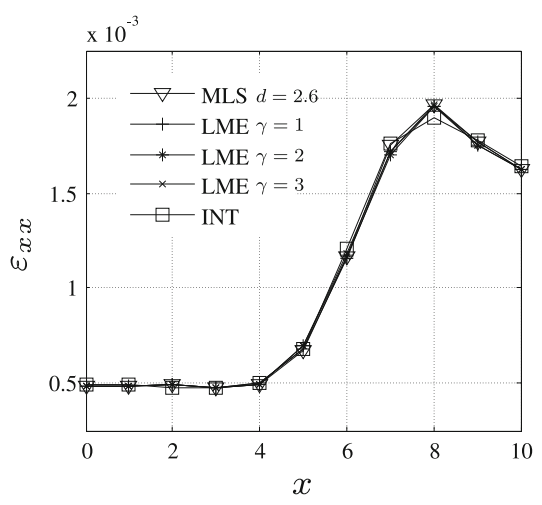

(c) SCNI integration

Fig. 13 Strain in $x$ direction along the symmetry axis for various settings of $\gamma$

approaching the reference finite element solution quite well for the small number of nodes.

If the number of volumetric constraints is reduced by selecting a lower order integration rule, results as shown in Fig. $12 \mathrm{~b}$ are obtained. For linear interpolation (INT), selecting either a three point integration rule, or a one point integration rule does not affect the results. For the LME and MLS approximations the scheme is responding spuriously due to the reduced integration. With the chosen parameters to control the domain of influence of these functions, a one point integration rule is insufficient.

Figure $12 \mathrm{c}$ shows the results for the nodally integrated approximations. It can be seen that independent of the shape function used, an accurate prediction of the strain is obtained. Even the linear interpolation, known for its poor behavior in incompressibility in the finite element method, is giving good results. At the point $x=8$ there is a minor overestimation of the strain but in general a good agreement is obtained. Furthermore, despite the use of diffuse shape functions, a distinct transition from the elastic region to the plastic region is predicted.

\subsubsection{Influence of compactness}

As shown in Fig. 12a, the MLS and LME functions give locking free results whereas the INT function shows locking when integrated with a standard integration rule. By setting the $\gamma$ parameter to a high or a low value, both the INT and MLS functions can be approximated respectively. Hence it is possible to move from the locking behavior of the INT function to the non-locking result of the MLS function by setting $\gamma$. To examine this effect the tapered bar problem is analyzed for three different settings of $\gamma$. These settings are $\gamma=1, \gamma=2$ and $\gamma=3$. Figure 13a shows the shape functions of the node lying on the symmetry axis at the location $(x, y)=(5,0)$. It can be seen that the local maximum-entropy shape function changes shape from the moving least-squares function to the interpolation by increasing $\gamma$. Figure 13 shows the result of the analysis for the two integration schemes. Indeed, Fig. 13b shows that the functions can be moved out of the locking domain by decreasing the compactness. A very similar effect was observed in case of the element-free Galerkin method by Dolbow and Belytschko [17] and by Askes et al. 
Fig. 14 Contour plot of the equivalent plastic strain
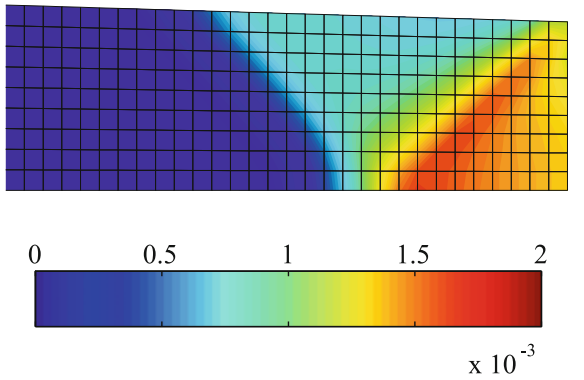

(a) FEM quad4 SRI
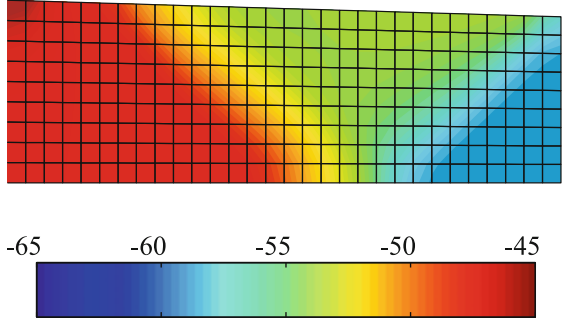

(a) FEM quad4 SRI

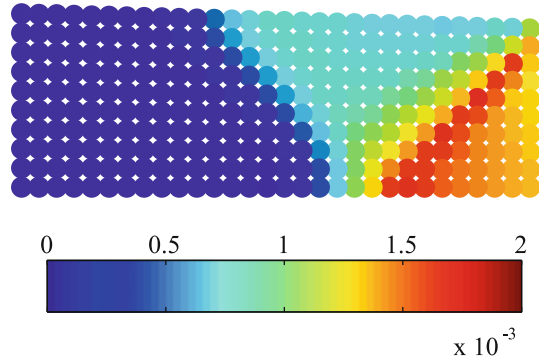

(b) $\mathrm{INT}+\mathrm{SCNI}$

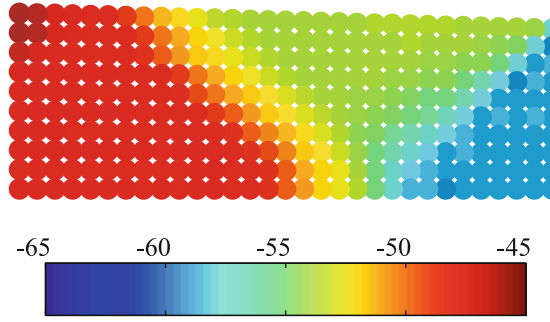

(b) $\mathrm{INT}+\mathrm{SCNI}$
[3]. In Fig. 13c the results of the same test are given but now for the nodal integration scheme. No effect of the compactness on the results is found. There is no locking, nor is there influence of the compactness of the approximation on the distribution of the strain. The nodal integration scheme gives very robust results for all shape functions.

\subsubsection{Full field results}

As shown in the previous analysis, the nodal integration scheme gives an accurate prediction for the strain on the symmetry line. In the following analysis the strain is examined outside this symmetry line by presenting contour plots of the equivalent plastic strain. Furthermore, the distribution of the pressure in the domain is investigated as well. For an accurate prediction of the elastic part of the deformation, this pressure should be physical and without numerical artifacts.

For the meshless computations, the INT function in combination with the SCNI integration scheme is used. A finite element computation is done for reference purposes. Both simulations use the same nodal grid, which has 10 nodes over the height and 31 nodes along the length, as shown in Fig. 14.

The finite element result and the meshless result are plotted in Fig. 14a and b, respectively. The equivalent plastic strain of the nodal integration scheme is plotted directly on the nodes. The integration point values of the finite element solution are presented in a contour plot without using nodal averaging. In general it can be seen that the two distributions correspond to good extend. An accurate prediction of the equivalent plastic strain is obtained with the nodal integration scheme and the linear triangular interpolation.
Secondly, the distribution of the pressure is investigated. The results for the two simulations are shown in Fig. 15. Again the patterns are found to be similar for the two simulations. Although the quadrilateral elements with selective reduced integration can suffer from pressure oscillations, these are not observed for this problem. The nodal integration scheme is showing very small oscillations in the plastic regime at the right-hand side of the bar. In the following section the inf-sup test will be performed in order to examine these oscillations and the locking-free behavior.

\subsection{The inf-sup test}

For the successful application of an approximation in incompressibility, the inf-sup test [or Ladyzhenskaya-BabuškaBrezzi (LBB) test] should be satisfied. The main formulation of this test can be found for instance in Chapelle and Bathe [11] Bathe [4].

For Gaussian integrated solutions, inf-sup tests can be found in the literature. For instance a Gaussian integrated triangle interpolation, is known to fail the inf-sup test, as is shown in [11]. For moving-least squares approximations Dolbow and Belytschko [17] reported on monitoring the infsup value. The approximation appeared to be locking. In the paper of Huerta et al. [19] a modification of the MLS function is proposed in order to satisfy the inf-sup test. This method is called the pseudo-divergence-free EFG method. Since the LME approximation is performing similar to the MLS function, no differences for the inf-sup test are expected.

For nodal integrated solutions locking free responses have been reported, though they have not been confirmed by a numerical inf-sup test. In this section, the locking free 
response as observed in the tapered bar problem, will be examined.

\subsubsection{General formulations}

First, two matrices $\mathbf{S}_{\mathrm{p}}$ and $\mathbf{S}_{\mathrm{u}}$ are defined, which correspond to the norm on the pressure field and the displacement field, respectively:

$$
\begin{aligned}
& \|\mathbf{u}\|^{2}=\int_{\Omega}(\tilde{\nabla} \mathbf{u})^{2} \mathrm{~d} \Omega=\mathbf{d}^{\mathrm{T}} \mathbf{S}_{\mathrm{u}} \mathbf{d} \\
& \|\mathbf{p}\|^{2}=\int_{\Omega} \mathbf{p}^{2} \mathrm{~d} \Omega=\mathbf{P}^{\mathrm{T}} \mathbf{S}_{\mathrm{p}} \mathbf{P}
\end{aligned}
$$

Vectors $\mathbf{d}$ and $\mathbf{P}$ contain the displacement and pressure degrees of freedom, respectively. The pressure norm expressed in displacement degrees of freedom is expressed as:

$$
\mathbf{G}=\mathbf{K}_{\mathrm{up}} \mathbf{S}_{\mathrm{p}} \mathbf{K}_{\mathrm{up}}^{\mathrm{T}}
$$

where matrix $\mathbf{K}_{\text {up }}$ is defined as:

$$
\mathbf{K}_{\text {up }}=\int_{\Omega} \tilde{\mathbf{B}}^{\mathrm{T}} \mathbf{N}_{\mathrm{p}} \mathrm{d} \Omega
$$

Vector $\mathbf{N}_{\mathrm{p}}$ contains the shape functions for the pressure space. For the SCNI a constant pressure is defined within a cell. $\mathbf{N}_{\mathrm{p}}$ is therefore simply 1 .

The numerical inf-sup condition as proposed by Chapelle and Bathe is given by [11]:

$$
\inf _{\mathbf{W}} \sup _{\mathbf{V}} \frac{\mathbf{W G V}}{\sqrt{\mathbf{W G W}} \sqrt{\mathbf{V S} \mathbf{V}}}=\psi>0
$$

where $\psi$ is a parameter that should be bounded away from zero in order to satisfy the condition and $\mathbf{W}$ and $\mathbf{V}$ are nodal displacement vectors. This $\psi$ can be found by solving the following eigen problem:

$\mathbf{G V}=\lambda \mathbf{S}_{\mathrm{u}} \mathbf{V}$

and taking the square root of the smallest non-zero eigenvalue in vector $\lambda$ :

$\psi=\sqrt{\lambda_{k}}$

The index $k$ of this smallest non-zero eigenvalue in vector $\lambda$ can be used to determine the number of spurious pressure oscillations. If $n_{\mathrm{u}}$ and $n_{\mathrm{p}}$ are the numbers of degrees of freedom in the displacement and pressure vectors respectively, then the number of oscillations $k_{\mathrm{pm}}$ is found by evaluating:

$k_{\mathrm{pm}}=k-\left(n_{\mathrm{u}}-n_{\mathrm{p}}+1\right)$

\subsubsection{Applying displacement boundary conditions}

To perform the numerical inf-sup test, a set of displacement degrees of freedom have to be prescribed. However, two of the shape functions do not posses the Kronecker delta property. To avoid adding degrees of freedom in case of the Lagrangian multipliers, the nodal displacements will be mapped on the field displacements with a strategy as proposed by Chen et al. [12]. The field displacements can be prescribed directly on the system by row-reduction techniques.

A mapping matrix $\mathbf{R}$ is constructed by sub-matrices $\boldsymbol{\Phi}_{i j}$ which are defined as follows:

$\mathbf{R}=\left[\begin{array}{cccc}\boldsymbol{\Phi}_{11} & \boldsymbol{\Phi}_{12} & \ldots & \boldsymbol{\Phi}_{1 N} \\ \boldsymbol{\Phi}_{21} & \boldsymbol{\Phi}_{22} & \ldots & \boldsymbol{\Phi}_{2 N} \\ \vdots & \vdots & \ddots & \vdots \\ \boldsymbol{\Phi}_{N 1} & \boldsymbol{\Phi}_{N 2} & \ldots & \boldsymbol{\Phi}_{N N}\end{array}\right]$

where:

$\boldsymbol{\Phi}_{i j}=\left[\begin{array}{cc}\phi_{j}\left(\mathbf{x}_{i}\right) & 0 \\ 0 & \phi_{j}\left(\mathbf{x}_{i}\right)\end{array}\right]$

The nodal displacements can be mapped to the field displacements of the nodes by the following equation:

$\mathbf{U}=\mathbf{R d}$

where $\mathbf{U}$ are the field displacements at the locations of the nodes and $\mathbf{d}$ are the nodal displacement degrees of freedom. Matrix $\mathbf{K}_{\text {up }}$ is converted to the field degrees of freedom as follows:

$\hat{\mathbf{K}}_{\text {up }}=\mathbf{R}^{-\mathrm{T}} \mathbf{K}_{\text {up }}$

Boundary displacements can be directly prescribed by rowreduction techniques on matrix $\hat{\mathbf{K}}_{\mathrm{up}}$. For matrix $\mathbf{S}_{\mathrm{u}}$ a similar approach is taken:

$\hat{\mathbf{S}}_{\mathrm{u}}=\mathbf{R}^{-\mathrm{T}} \mathbf{S}_{\mathrm{u}} \mathbf{R}^{-1}$

\subsubsection{Numerical results}

Figure 16 shows the problem as proposed by Chapelle and Bathe [11]. Figure 17 shows the node grids as used for the test. Both regular grids and irregular grids of various densities will be used.

The results of the inf-sup test on the regular grid are given in Fig. 18a. Similarly, the results of the irregular grid are given in Fig. 18b. It can be seen that for the regular grid as well as the irregular grid the inf-sup test is passed. The $\psi$-parameter is bounded away from zero and does not decrease on nodal grids with increasing density. Based on this test it can be concluded that matrix $\mathbf{K}_{\text {up }}$ does not over-constrain the system of equations. Volumetric locking is likely to be absent based on the results of this test. Although locking 


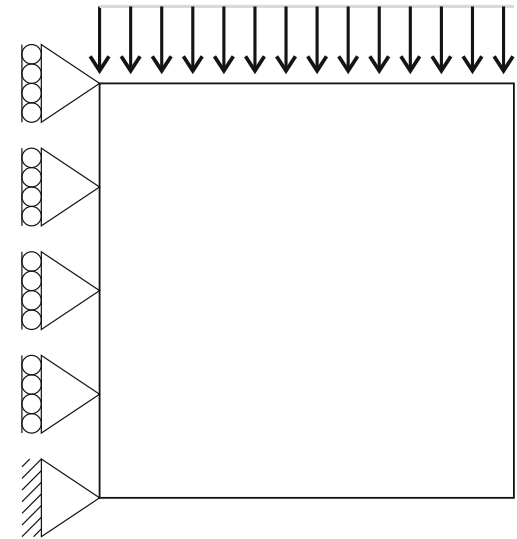

Fig. 16 Model for the inf-sup test

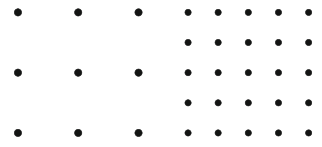

(a) $3 \times 3$

(b) $5 \times 5$

(c) $9 \times 9$

(d) $17 \times 17$
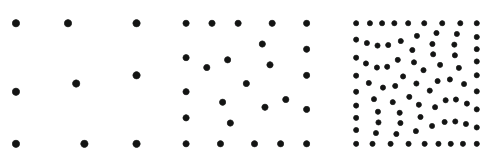

(f) $3 \times 3$

(g) $5 \times 5$

(h) $9 \times 9$

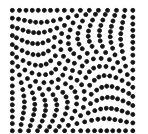

(i) $17 \times 17$

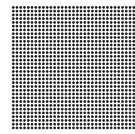

(e) $33 \times 33$

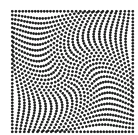

(j) $33 \times 33$
Fig. 17 Nodes sets for the inf-sup test. Figures a-e show the regular grids. Figures $\mathbf{f}-\mathbf{j}$ show the irregular grids

appears to be not an issue for the SCNI integration, spurious oscillations are of concern. For all the regular grids, a rank deficiency of 1 on matrix $\mathbf{K}_{\text {up }}$ is detected for the diffuse approximations. The same holds for the triangular interpolation, although here two eigenvalues are zero. Figure 19 shows one of the detected checkerboard patterns in the pressure field for the INT function. For the irregular grid, no rank deficiency was detected of matrix $\mathbf{K}_{\text {up }}$. Nevertheless, for instance the same oscillation as shown in Fig. 19 is present though its corresponding eigenvalue is not zero. To conclude, matrix $\mathbf{K}_{\text {up }}$ appears not to be over-constraining the system, though stable pressure fields cannot be guaranteed.

\subsection{Computational efficiency}

In the following test, the computational efficiency of the components as shown in Fig. 3 is investigated. To examine this efficiency, two aspects of the code will be monitored. The first aspect is the time to build the stiffness matrix and the second is the required memory allocation for this matrix. The plate with a hole problem with the cloud of nodes as shown in Fig. $6 \mathrm{~b}$ is used for this test.

The time to build the stiffness matrix includes the computations of the shape functions, setting-up of the integration rule and assembly of all local stiffness matrices in the global stiffness matrix. These local stiffness matrices are con-

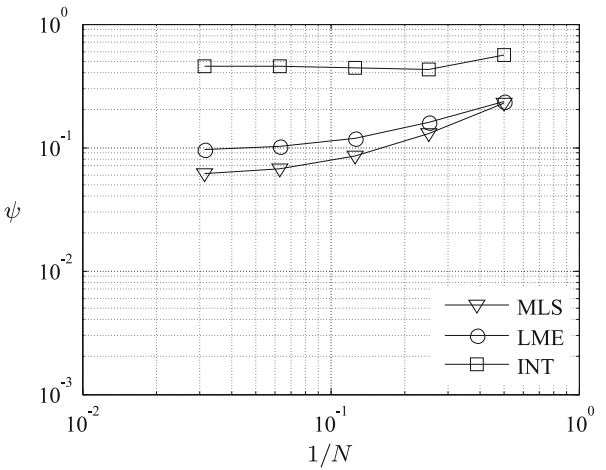

(a) regular grid, SCNI integration

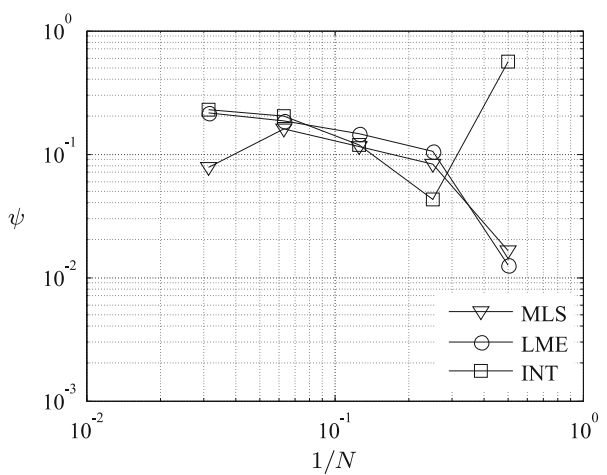

(b) irregular grid, SCNI integration

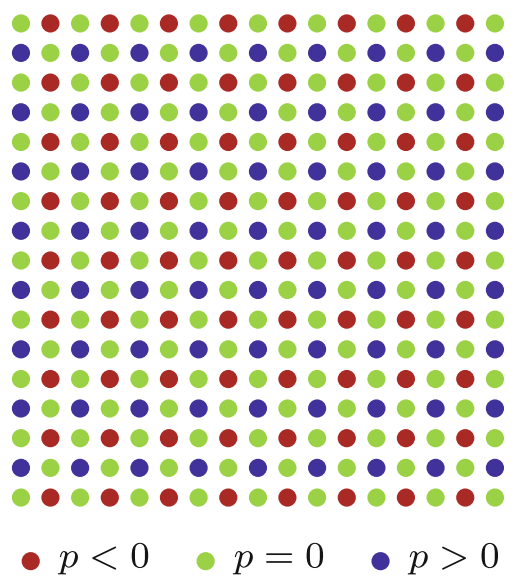

Fig. 19 Pressure oscillation as detected on the regular grid

structed per cell or per triangle depending on whether the nodal integration scheme or the Gaussian scheme is selected, respectively. The time does not include the neighbor search as it is required for the MLS and LME shape functions, nor is the time for the construction of the Lagrangian multipliers or performing the triangulation or tesselation included. These components were found to be of small influence on the total computation time for this set of nodes. The meshless code is programmed in MATLAB and the simulations are performed on a dual-core $2.3 \mathrm{GHz}$ computer. Although computational 
Table 1 Time for constructing $\mathbf{K}$ for the plate with hole problem in seconds

\begin{tabular}{llll}
\hline & MLS & LME & INT \\
\hline STD & 4.589 & 4.124 & 0.307 \\
SCNI & 7.268 & 6.099 & 0.909 \\
\hline
\end{tabular}

Table 2 Number of non-zeros in $\mathbf{K}$ for the plate with hole problem

\begin{tabular}{llll}
\hline & MLS & LME & INT \\
\hline STD & 30,028 & 26,476 & 2,802 \\
SCNI & 49,064 & 53,500 & 9,299 \\
\hline
\end{tabular}

times of a computer code are depending on many factors, for instance the skill of the programmer, the trends of the analysis are expected to be representative.

Table 1 shows the results for the shape functions and integration schemes in seconds. Several trends can be observed in the table. Firstly, it can be seen that the MLS and LME shape function are considerably more involving than the simple INT approximation. This could be expected since the INT function requires only a few computations in order to obtain $\phi$ and the local stiffness matrices of this function are considerably smaller in size. Therefore less multiplications are performed in the assembly procedure. Secondly, the SCNI integration scheme is computationally more demanding than the STD integration. For both integration schemes, constructing the tesselation and the triangulation is left out of the analysis. Therefore it can be concluded that the assembly procedure and the construction of the assumed gradient makes the SCNI integration more involving. The extra computational effort is of little importance for the diffuse approximations. For the INT approximation however, the type of integration is of more influence on the computation time relatively.

Table 2 shows the number of non-zero elements in the stiffness matrix for the shape functions and the integration schemes. The total size of the stiffness matrix $\mathbf{K}$ is 496 by 496. The stiffness matrix does not include the Lagrangian multiplier matrices $\mathbf{G}$ and furthermore the stiffness matrix $\mathbf{K}$ is defined symmetric and sparse. The table shows that the memory allocation for the INT shape function is less than for the diffuse shape functions. The diffuse character of the LME and MLS approximations has a big influence on the sparseness of the stiffness matrix. Furthermore the number of non-zero elements in $\mathbf{K}$ increases by employing the stabilized nodal integration. Due to the compact character of the INT shape function this effect is relatively more pronounced than it is for the other two shape functions. It can be expected that this will also influence the solution time of the system of equations, especially if the models are large.

Two remarks have to be made on the results of the timings. Solving the system of equations, the triangulations and the neighbor search routine were found to have a negligible influence on the total computation time. These parts of the algorithm scale more than linear with the number of nodes and therefore their relative contribution to the total computation time will increase compared to the building time of the stiffness matrix. Secondly, in the case of geometrical or material non-linearity, shape functions are required per iteration to build the stiffness matrix and the internal force vector. By storing the shape functions and 're-using' them over the increment, the relative cost of the more involving shape functions can be reduced. For the MLS and LME functions this can be a time reducing approach. The difference between the linear interpolation and the MLS and LME approximations as shown in Table 1 is expected to be smaller in that case. A drawback of this implementation strategy is that memory has to be allocated for the gradients of the shape functions.

\section{Conclusions}

In this paper a numerical analysis was performed on three meshless approximations and two integration schemes. The performance in linear elasticity and in elasto-plasticity was investigated.

It was shown that diffuse shape functions, like the moving least-squares function and the local maximum-entropy function offer a better accuracy when compared to the linear triangle interpolation in elasticity. The error reduced approximately with a factor of two to three when using the MLS or LME approximation instead of a linear triangle interpolation. However, the computational effort for these two diffuse approximations is higher. The stiffness matrix becomes less sparse and the time for building this matrix is higher. The local maximum-entropy approximation and the moving least-squares approximation were found to perform very similar if used with the same domain of influence. Furthermore it was observed that the SCNI integration scheme is less sensitive to distortion than the Gaussian integration scheme.

A test in elasto-plasticity showed that using a Gaussian integration rule gives results that are strongly depending on the order of the integration rule and the compactness of the diffuse approximations. Both spurious deformation modes or volumetric locking can be present, depending on the choices in the integration rule or the compactness of the shape function. The SCNI integration scheme on the contrary, gives excellent results when compared to a Gaussian integration rule. No trace of volumetric locking is observed. The meshless solutions match the reference solution accurately and the results were found to be nearly independent of the type of shape function used. Moreover, the compactness of the shape function has a negligible influence on the results. To give a more fundamental background to the observed locking-free response of the SCNI integration, the numerical inf-sup test 
was performed. The SCNI integration appears locking free, however spurious oscillations were detected. Stabilization of this oscillation is the authors current effort. To conclude, it can be stated that the SCNI integration is the most sensible option for use in combination with incompressible material models, leaving the choice of shape function open. Simply using a Gaussian integration scheme is proving to be not optimal for use in incompressibility. For these cases, the extra computational effort for the SCNI integration is easily justified. For cases in which highly accurate and mesh independent results are required, the diffuse approximations can be an interesting option. However, for a robust and fast simulation, the method of SCNI in combination with triangle interpolation (SFEM) shows to be a very efficient computational scheme.

Acknowledgments This research was carried out under project number MC1.07291 in the framework of the Research Program of the Materials innovation institute M2i (http://www.m2i.nl), the former Netherlands Institute for Metals Research.

Open Access This article is distributed under the terms of the Creative Commons Attribution Noncommercial License which permits any noncommercial use, distribution, and reproduction in any medium, provided the original author(s) and source are credited.

\section{References}

1. Alfaro I, Bel D, Cueto E, Doblaré M, Chinesta F (2006) Threedimensional simulation of aluminium extrusion by the $\alpha$-shape based natural element method. Comput Methods Appl Mech Eng 195:4269-4286

2. Arroyo M, Ortiz M (2006) Local maximum-entropy approximation schemes: a seamless bridge between finite elements and meshfree methods. Int J Numer Methods Eng 65:2167-2202

3. Askes H, de Borst R, Heeres O (1999) Conditions for locking-free elasto-plastic analysis in the element-free galerkin method. Comput Methods Appl Mech Eng 173:99-109

4. Bathe KJ (2006) Finite element procedures. Klaus-Jürgen Bathe

5. Belytschko T, Guo Y, Liu WK, Xiao SP (2000) A unified stability analysis of meshless particle methods. Int J Numer Methods Mech Eng 48:1359-1400

6. Belytschko T, Krongauz Y, Organ D, Fleming M, Krysl P (1996) Meshless methods: an overview and recent developments. Comput Methods Appl Mech Eng 139(1-4):3-47

7. Belytschko T, Krysl P, Krongauz Y (1997) A three dimensional explicit element-free galerkin method. Int J Numer Methods Fluids 24:1253-1270

8. Belytschko T, Lu YY, Gu L (1994) Element-free galerkin methods. Int J Numer Methods Eng 37:229-256

9. Bonet J, Burton AJ (1998) A simple average nodal pressure tetrahedral element for incompressible and nearly incompressible dynamic explicit applications. Commun Numer Methods Eng 14:437-449

10. Braun J, Sambridge M (1995) A numerical method for solving partial differential equations on highly irregular evolving grids. Nature 376:655-660

11. Chapelle D, Bathe KJ (1993) The inf-sup test. Comput Struct 47:537-545
12. Chen JS, Pan C, Wu CT, Liu WK (1996) Reproducing kernel particle methods for large deformation analysis of non-linear structures. Comput Methods Appl Mech Eng 139:195-227

13. Chen JS, Wu CT, Yoon S, You Y (2001) A stabilized conforming nodal integration for galerkin mesh-free methods. Int J Numer Methods Eng 50:435-466

14. Chen JS, Yoon S, Wu CT (2002) Non-linear version of stabilized conforming nodal integration for galerkin mesh-free methods. Int J Numer Methods Eng 53:2587-2615

15. Dohrmann CR, Heinstein MW, Jung J, Key SW, Witkowski WR (2000) Node-based uniform strain elements for three-node triangular and four-node tetrahedral meshes. Int J Numer Methods Eng 47:1549-1568

16. Dolbow J, Belytschko T (1999) Numerical integration of the galerkin weak form in meshfree methods. Comput Mech 23: 219-230

17. Dolbow J, Belytschko T (1999) Volumetric locking in the element free galerkin method. Int $\mathrm{J}$ Numer Methods Eng 46(6):925-942

18. González D, Cueto E, Martínez MA, Doblaré M (2004) Numerical integration in natural neighbour galerkin methods. Int J Numer Methods Eng 60:2077-2104

19. Huerta A, Vidal Y, Villon P (2004) Pseudo-divergence-free element-free galerkin method for incompressible fluid flow. Comput Methods Appl Mech Eng 193:1119-1136

20. Hung NX, Bordas SPA, Hung ND (2009) Adressing volumetric locking and instabilities by selective integration in smoothed finite elements. Commun Numer Methods Eng 25:19-34

21. Idelsohn SR, Oñate E (2006) To mesh or not to mesh. that is the question. Comput Methods Appl Mech Eng 195:4681-4696

22. Idelsohn SR, Oñate E, Calvo N, Pin FD (2003) The meshless finite element method. Int J Numer Methods in Eng 58:893-912

23. Krysl P, Zhu B (2008) Locking-free continuum displacement finite elements with nodal integration. Int $\mathrm{J}$ Numer Methods Eng 76:1020-1043

24. Li S, Liu WK (2002) Meshfree and particle methods and their applications. Appl Mech Rev 55(1):1-34

25. Liu GR, Gu YT (2001) A point interpolation method for twodimensional solids. Int J Numer Methods Eng 50:937-951

26. Liu GR, Nguyen TT, Dai KY, Lam KY (2007) Theoretical aspects of the smoothed finite element method (sfem). Int J Numer Methods Eng 71:902-930

27. Liu GR, Nguyen-Thoi T, Nguyen-Xuan H, Lam KY (2009) A node-based smoothed finite element method (ns-fem) for upper bound solutions to solid mechanics problems. Comput Struct $87: 14-26$

28. Liu WK, Chen Y, Jun S, Chen JS, Belytschko T, Pan C, Uras RA, Chang CT (1996) Overview and applications of the reproducing kernel particle methods. Arch Comput Methods Eng 3:3-80

29. Liu WK, Han W, Lu H, Li S, Cao J (2004) Reproducing kernel element method. Part I. Theoretical formulation. Comput Methods Appl Mech Eng 193:933-951

30. Liu WK, Jun S, Li S, Adee J, Belytschko T (1995) Reproducing kernel particle methods for structural dynamics. Int J Numer Methods Eng 38:1655-1679

31. Liu WK, Jun S, Zhang YF (1995) Reproducing kernel particle methods. Int J Numer Methods Fluids 20:1081-1106

32. Liu WK, Li S, Belytschko T (1997) Moving least-square reproducing kernel methods (i) methodology and convergence. Comput Methods Appl Mech Eng 143:113-154

33. Lucy LB (1977) A numerical aproach to the testing of the fission hypothesis. Astron J 82:1013-1024

34. Nayroles B, Touzot G, Villon P (1992) Generalizing the finite element method: diffuse approximation and diffuse elements. Comput Mech 10:307-318 
35. Pannachet T, Askes H (2000) Some observations on the enforcement of constraint equations in the efg method. Commun Numer Methods Eng 16:819-830

36. Pires FMA, de Souza Neto EA, de la Cuesta Padilla JL (2004) An assessment of the average nodal volume formulation for the analysis of nearly incompressible solids under finite strains. Commun Numer Methods Eng 20:569-583

37. Puso MA, Chen JS, Zywicz E, Elmer W (2008) Meshfree and finite element nodal integration methods. Int J Numer Methods Eng 74:416-446

38. Puso MA, Solberg J (2006) A stabilized nodally integrated tetrahedral. Int J Numer Methods Eng 67(6):841-867

39. Simo JC, Hughes TJR (1998) Computational inelasticity, interdisciplinary applied mathematics, vol 7. Springer, Berlin

40. Sukumar N (2004) Construction of polygonal interpolants: a maximum entropy approach. Int $\mathrm{J}$ Numer Methods Engineering 61:2159-2181
41. Sukumar N, Wright RW (2007) Overview and construction of meshfree basis functions: from moving least squares to entropy approximants. Int J Numer Methods Eng 70:181-205

42. Timoshenko S, Goodier JN (1951) Theory of elasticity. McGraw, New York

43. Wang JG, Liu GR (2002) A point interpolation meshless method based on radial basis functions. Int $\mathrm{J}$ Numer Methods Eng 54: $1623-1648$

44. Yoo JW, Moran B, Chen JS (2004) Stabilized conforming nodal integration in the natural-element method. Int J Numer Methods Eng 60:861-890

45. Zienkiewicz OC, Taylor RL (2000) The finite element method, vol 1. The basis, 5 edn. Butterworth-Heinemann, Oxford 